\title{
Enhanced Fluorescence from Fluorophores on Fractal Silver Surfaces
}

\author{
Alexandr Parfenov ${ }^{\dagger}$, Ignacy Gryczynski ${ }^{\dagger}$, Joanna Malicka ${ }^{\dagger}$, Chris D. Geddes, ${ }^{*, *}$ and \\ Joseph R. Lakowicz ${ }^{*, \dagger}$
}

Center for Fluorescence Spectroscopy, University of Maryland at Baltimore, Department of Biochemistry and Molecular Biology, 725 West Lombard Street, Baltimore, Maryland 21201, and Institute of Fluorescence and Center for Fluorescence Spectroscopy, Medical Biotechnology Center, University of Maryland Biotechnology Institute, 725 West Lombard Street, Baltimore, Maryland 21201

Received: December 31, 2002; In Final Form: May 22, 2003

\begin{abstract}
Recent reports have shown enhanced fluorescence for fluorophores in close proximity to chemically deposited silver islands or colloids. To expand the usefulness of metal-enhanced fluorescence we tested fractal silver structures formed on, or near, silver electrodes by passage of electric currents. The emission intensity of fluorescein-labeled human serum albumin (FITC-HSA) was enhanced over 100-fold when adsorbed to the fractal silver structures as compared to glass. The amplitude-weighted lifetime is dramatically reduced to near 3 ps. Enhanced fluorescence was shown to result in selective observation of FITC-HSA over a fluorophore not attached to the silver surface. And finally, photostability measurements indicate 160-fold more photons are detectable from FITC-HSA on the fractal silver surface. These results suggest the use of in situ generated silver structures for metal-enhanced fluorescence.
\end{abstract}

\section{Introduction}

In several recent reports we described the favorable effects of silver particles on fluorophores near the metallic surfaces. ${ }^{1-4}$ These effects include increased quantum yields, decreased lifetimes, increased photostability, and increased energy transfer. These effects are due to interactions of the excited-state fluorophores with the surface plasmon resonances on the metallic surfaces..$^{5-8}$ These interactions of the fluorophore with the metal surface can result in increased rates of excitation, quenching, increased intensities, and/or increased quantum yield. For our purposes of using these interactions to obtain enhanced fluorescence, the most important effect is an increase in the radiative decay rate for fluorophores which occurs at $\approx 50-$ $100 \AA$ from the metallic surface. We refer to the use of fluorophore-metal interactions as radiative decay engineering. ${ }^{9}$

In previous reports on this topic we used silver island films, which are subwavelength-size silver particles deposited on glass by reduction of silver nitrate. We also examined colloids which were also made by chemical reduction. ${ }^{10}$ In many applications of fluorescence it would be advantageous to have localized silver deposition for spatially selective analysis, such as lab-on-a-chip systems. ${ }^{11-13}$ Localized silver colloid formation has been accomplished with reagents in laminar flow, ${ }^{14}$ by nanolithography ${ }^{15,16}$ and by electroplating on insulators. ${ }^{17} \mathrm{We}$ reasoned that metallic silver particles of appropriate size should be effective, irrespective of the mode of deposition. Additionally, we were aware of the extensive use of roughened silver electrodes for surface-enhanced Raman scattering (SERS). ${ }^{18-20}$ Hence we examined silver electrodes following passage of electrical current. This treatment resulted in the formation of fractal-like structures as recently reported. ${ }^{17}$ These freshly generated silver surfaces were allowed to bind a protein human

* To whom correspondence should be addressed. E-mail: cfs@ cfs.umbi.umd.edu or chris@ @fs.umbi.umd.edu. Fax: (410) 7068408.

$\dagger$ University of Maryland at Baltimore, Department of Biochemistry and Molecular Biology.

$\stackrel{\doteqdot}{\doteqdot}$ University of Maryland Biotechnology Institute.
SCHEME 1: Configuration for Creation of Fractal Silver Surfaces on Silver Electrodes and Glass

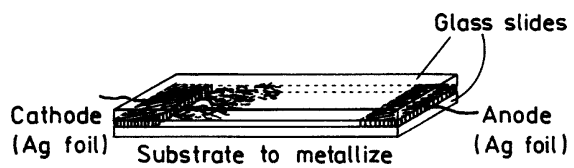

serum albumin (HSA) which had been heavily labeled with fluorescein isothiocyanate (FITC), resulting in self-quenching of the fluorescein. We found the intensities of FITC-HSA were increased over 100-fold when bound to these structures.

\section{Materials and Methods}

Fractal-like silver structures were generated by using two silver electrodes held between microscope slides (Scheme 1). The silver electrodes were $10 \times 35 \times 0.1 \mathrm{~mm}$, with about 20 $\mathrm{mm}$ between the two electrodes. Deionized water was placed between the slides. A direct current of $100 \mu \mathrm{A}$ was passed between the electrodes for about $10 \mathrm{~min}$, during which the voltage started near $5 \mathrm{~V}$ and decreased to $2 \mathrm{~V}$. During the current flow fractal silver nanostructures grew on the cathode and then on the glass near the cathodes.

The silver structures were observed using the apparatus shown in Scheme 2. Bright field and fluorescence images were obtained with a Roper RLX-37 CCD camera. For fluorescence the 442$\mathrm{nm}$ excitation was obtained from a HeCd laser, using about 2 $\mathrm{mW}$. Emission was isolated with a 540-nm long-wave pass filter and a liquid cutoff filter at $525 \mathrm{~nm}$. The liquid filter was an aqueous solution of $\mathrm{CrO}_{4}{ }^{2-}$ and $\mathrm{Cr}_{2} \mathrm{O}_{7}{ }^{2-}$ which completely eliminated scattered light in lieftime measurements and did not display autofluorescene.

Human serum albumin (HSA) was from Sigma. Two milligrams of human serum albumin was dissolved in $1 \mathrm{~mL}$ of 0.1 $\mathrm{M}$ bicarbonate buffer ( $\mathrm{pH}$ 9.2) and was mixed with $70 \mu \mathrm{L}$ of fluorescein-5-isothiocyanate (FITC; Molecular Probes) solution in DMSO (2 $\mathrm{mg}$ of FITC/200 $\mu \mathrm{L}$ of DMSO). The reaction 


\section{SCHEME 2: Instrumentation Used for Observation of Silver Surfaces and FITC-HSA Fluorescence}

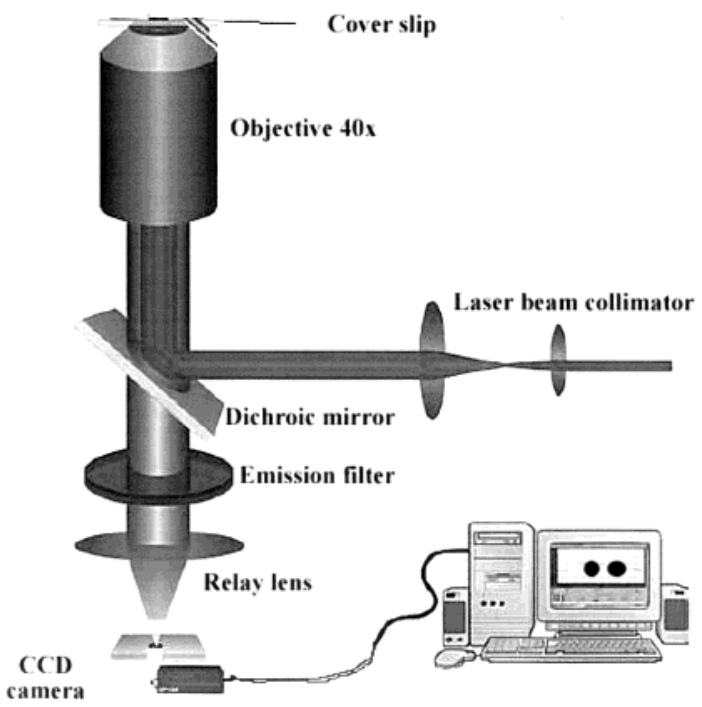

mixture was incubated for $2 \mathrm{~h}$ at room temperature and the labeled protein was separated from the unreacted probe by passing over a Sephadex G-25 column equilibrated with $0.1 \times$ PBS.

The ratio FITC/HSA in stock solution of labeled protein was determined by independent measurements of dye and protein concentrations, respectively. The amount of FITC was calculated using absorbance of FITC-HSA conjugates in $0.1 \mathrm{M}$ bicarbonate buffer ( $\mathrm{pH} 9.2$ ) at $495 \mathrm{~nm}$ and molar extinction coefficient of FITC $\epsilon(495 \mathrm{~nm})=76000 \mathrm{M}^{-1} \mathrm{~cm}^{-1}$. The HSA concentration was determined by using Coomassie Plus protein assay reagent (Pierce, IL). For fluorescence measurements we used a sample with an average FITC/HSA ratio of 7 (see further explanation later).

The FD intensity decays were analyzed in terms of the multiexponential model

$$
I(t)=\sum_{i} \alpha_{i} \exp \left(-t / \tau_{i}\right)
$$

where $\tau_{i}$ are the lifetimes with amplitudes $\alpha_{i}$ and $\Sigma \alpha_{i}=1.0$. The frequency domain instrumentation and fitting to the multiexponential model are both routine instrumentation and concepts and were performed as described previously. ${ }^{22-23}$ Excitation was at $514 \mathrm{~nm}$ from an argon ion laser, repetition rate of $76 \mathrm{MHz}$. Emission was observed using magic angle polarization conditions. The contribution of each component to the steady-state intensity is given by

$$
f_{i}=\frac{\alpha_{i} \tau_{i}}{\sum_{j} \alpha_{j} \tau_{j}}
$$

where the sum in the denominator is over all the decay times and amplitudes. The mean decay time is given by

$$
\bar{\tau}=\sum_{i} f_{i} \tau_{i}
$$

The amplitude-weighted lifetime is given by

$$
\langle\tau\rangle=\sum_{i} \alpha_{i} \tau_{i}
$$

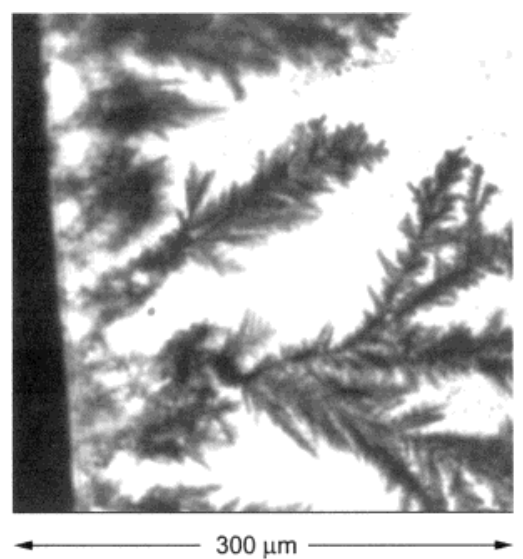

Figure 1. Silver nanostructures on, or near, the silver cathode treated with $\mathrm{SnCl}_{2}$. Bright field image.

For these measurements the background was less than $1 \%$ from an equivalent sample of silver coated with unlabeled HSA.

\section{Results}

We examined the silver electrodes during passage of electrical current. Upon starting the current, we observed rapid growth of fractal-like structure on the cathode (Figure 1). These structures grew rapidly and appeared to twist as they grew. After a short time the silver began to grow onto the glass slide (Figure 2 ). These structures are similar to those reported recently during electroplating of insulators. ${ }^{17} \mathrm{We}$ found that dipping the slides in $0.001 \mathrm{mg} / \mathrm{dL}$ of $\mathrm{SnCl}_{2}$ for $30 \mathrm{~min}$ prior to electrolysis resulted in structures which were firmly bound to the glass during working. Without $\mathrm{SnCl}_{2}$ similar nanostructures were formed but were partially removed during washing.

We examined the usefulness of these silver structures for metal-enhanced fluorescence (MEF). Following passage of current, the silvered slide was soaked in $10 \mu \mathrm{M}$ FITC-HSA overnight at $4{ }^{\circ} \mathrm{C}$, which is thought to result in a monolayer of surface-bound HSA. ${ }^{21,24}$ This sample of FITC-HSA contained approximately seven fluorescein molecules per HSA molecule, so that the fluorescein emission was partially quenched by resonance energy transfer (RET) between the fluorophores. ${ }^{21}$ Using partially self-quenched fluorescein also potentially affords for the "release" or "unquenching" of fluorescence, as the fluorescein is already self-quenched. These over-labeled proteins are ideal choices as probes, because the modified radiative decay rate near the metallic silver surface competes with quenching (a nonradiative process), in essence returning the quenched fluorescence signal. While our probe is somewhat exotic in its unquenching nature near silver, a similar effect is also observed for a single label, but at a reduced total intensity.

We measured a fluorescence image of a fractal silver surface using the same apparatus (Scheme 2) with 442-nm excitation (Figure 3). The fluorescence image of the area in Figure 2C shows a fluorescence pattern which closely follows the shape of the silver particles (Figure 3). Interestingly, regions of high and low fluorescence intensity were observed. This result is roughly consistent with recent SERS data which showed the presence of intense signals, which appeared to be located between clusters of particles. ${ }^{25-26}$ Emission spectra were collected from eight selected regions of varying brightness. In all cases, the emission spectra appeared to be that of fluorescein (Figure 4). In these spectra the blue edge of the fluorescein emission is cut off by the emission filter. As a blank control, the silver structures were coated with unlabeled HSA. The 
A

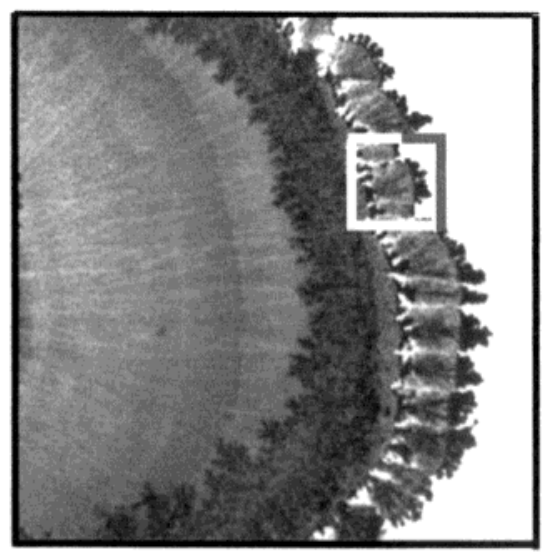

$500 \mu \mathrm{m}$
B

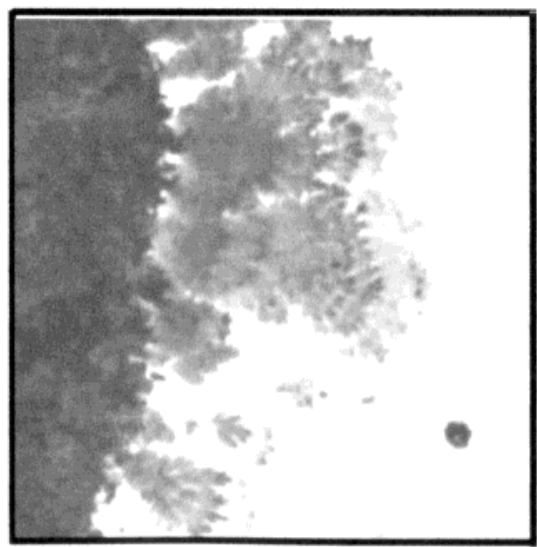

$60 \mu \mathrm{m}$
C

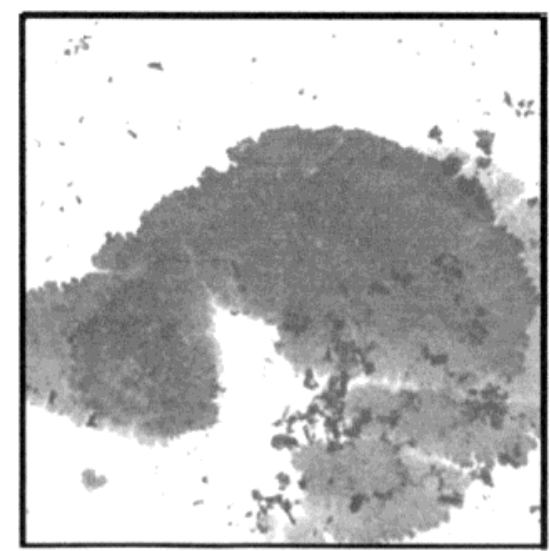

$20 \mu \mathrm{m}$

Figure 2. Silver nanostructures deposited on glass during electroplating (A). Panels B and C are consecutive magnifications of the marked area on panel A. Bright field image.

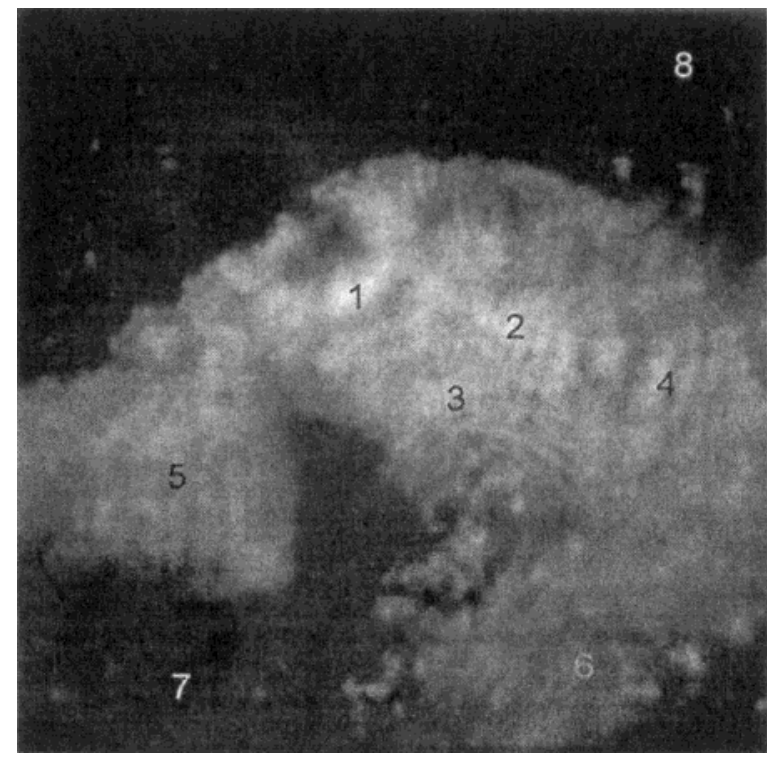

Figure 3. Fluorescence image of FITC-HSA deposited on the silver structure shown in Figure 2C.

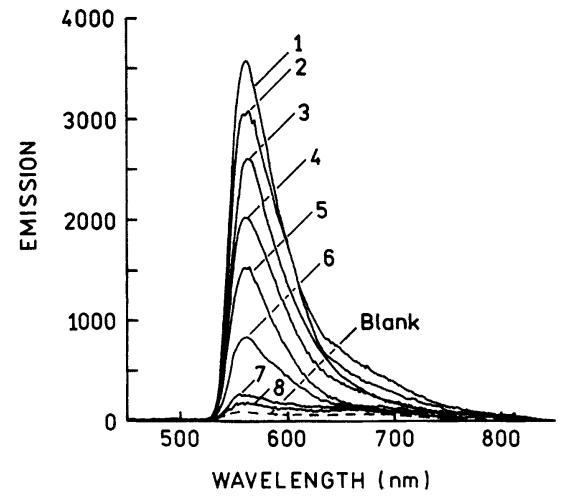

Figure 4. Emission spectra of the numbered areas marked in Figure 3.

resulting signal was lower than those of any of the silvered areas and lower than regions of the unsilvered glass treated with FITCHSA.

We spatially scanned the fluorescence emission intensity on a diagonal path across the silver structure (Figure 5). The
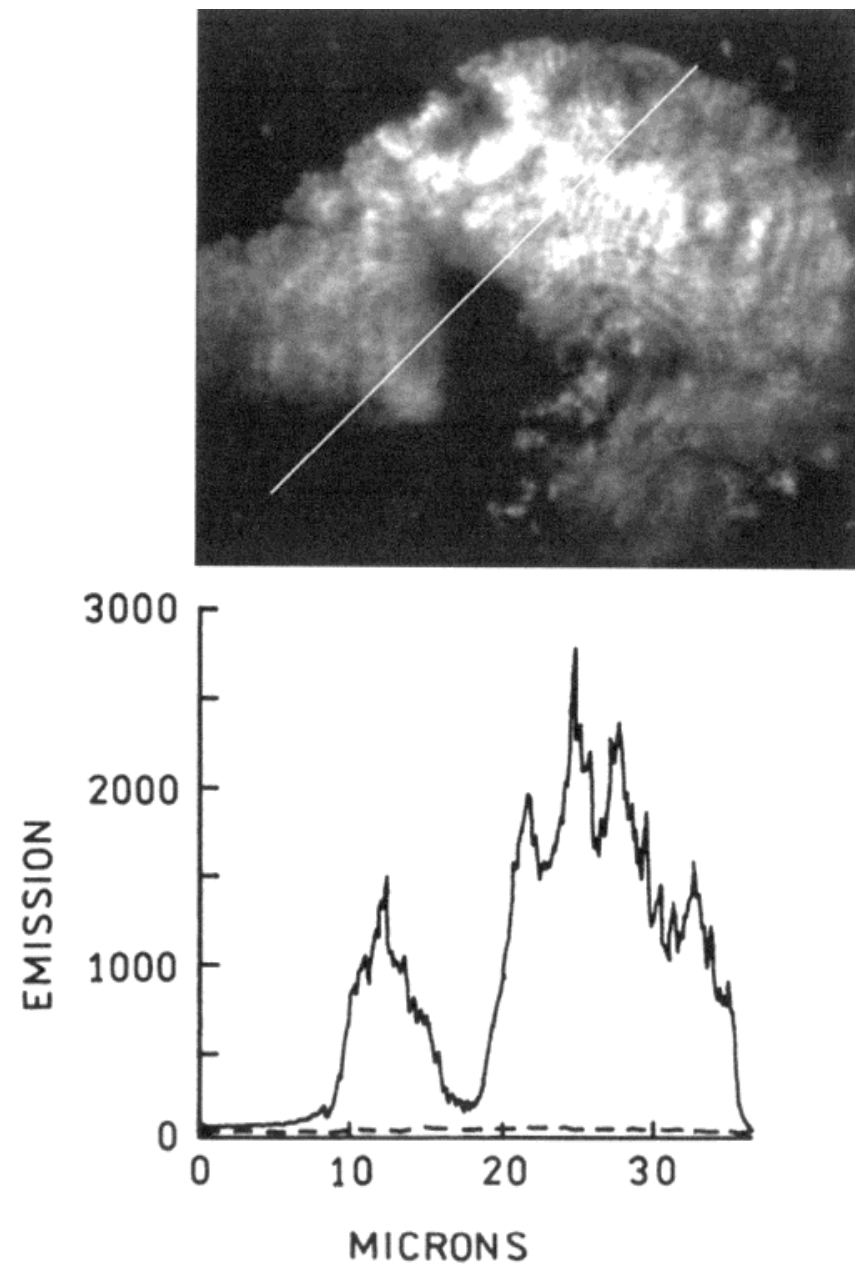

Figure 5. Diagonal scan of the emission intensity of FITC-HSA. The dashed line is the intensity observed across a line of equivalent length across unsilvered glass.

emission intensity varied roughly with the density of the deposited silver (Figure 2C). The lower dotted line in Figure 5 represents the intensity of FITC-HSA on unsilvered glass. Most of this signal appears to be due to dark counts in the CCD camera. The emission intensities range from 100-fold (position 6) to 600-fold (position 1) greater than the signal from 


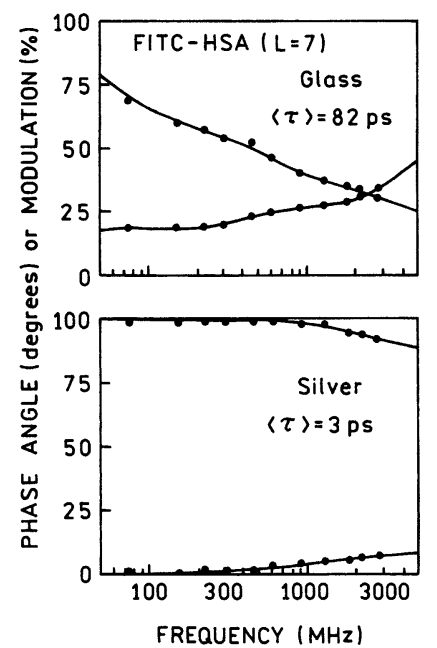

Figure 6. Frequency-domain intensity decays of FITC-HSA on glass (top) and deposited silver (bottom).

FITC-HSA on unsilvered glass. We recognized that some of this increase could be due to binding of more FITC-HSA to silver structures with large surface areas. We note that the fluorescein is not quenched on the surface, probably because the size of an HSA molecule positions the fluorescein about 40 $\AA$ from the surface, which is near the distance for maximal radiative rate and therefore fluorescence enhancement. ${ }^{27}$

There are probably several contributions to the increased intensities of FITC-HSA on the fractal silver surfaces. These causes include more protein binding, increased rates of excitation due to an enhanced electric field, and larger quantum yields due to increased rates of radiative decay. If the radiative decay rate is increased, then the lifetime should decrease. ${ }^{9} \mathrm{We}$ measured the frequency-domain intensity decays of FITC-HSA bound to unsilvered glass and fractal silver (Figure 6). The amplitude-weighted lifetime of FITC-HSA bound to glass is about $80 \mathrm{ps}$, in agreement with previous measurements of selfquenched fluorescein on HSA. ${ }^{21}$ On fractal silver the amplitudeweighted lifetime is dramatically reduced to about $3 \mathrm{ps}$. We carefully considered whether this decrease was due to detection of scattered light. The background signal from unlabeled HSA on fractal silver was less than $1 \%$, too quick to be resolved by our instrumentation. The emission filter combination of a 540$\mathrm{nm}$ interference filter and a solution of $\mathrm{CrO}_{4}{ }^{2-} / \mathrm{Cr}_{2} \mathrm{O}_{7}{ }^{2-}$ was selected for low emission from the filter when exposed to scattered light from the sample. We believe part of the increased intensity is due to release of fluorescein self-quenching due to the silver surface. ${ }^{21}$

We tested the use of fractal silver for providing emission selectively from fluorophores in close proximity to the surface. For this purpose we examined glass coated with FITC-HSA, to which we added a solution of nile blue, to yield an over 10fold larger signal from nile blue (Figure 7, top). In contrast to the glass surface, for the silver surface the signal was now dominated by the FITC-HSA (bottom). Intuitively, this result suggests that unwanted autofluorescence from biological samples can be avoided by metal-enhance fluorescence of surfacelocalized fluorophores.

And finally, we studied the photostabilities of FITC on the fractal silver surface, silver island films (SIFs), and uncoated quartz. While the relative photobleaching is higher on fractal silver, the increased rate of photobleaching is less than the increase in intensity (Figure 8). From the areas under these curves we estimate 16- and 160-fold more photons can be

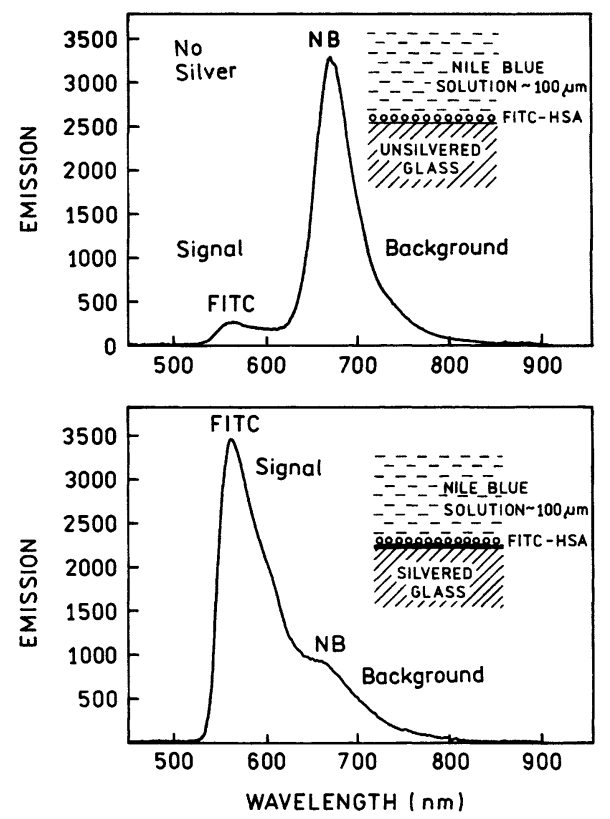

Figure 7. Emission spectra of a monolayer of FITC-HSA on glass (top) or silver (bottom) in the presence of $2 \times 10^{-6} \mathrm{M}$ nile blue. The sample was $100 \mu \mathrm{M}$ thick.

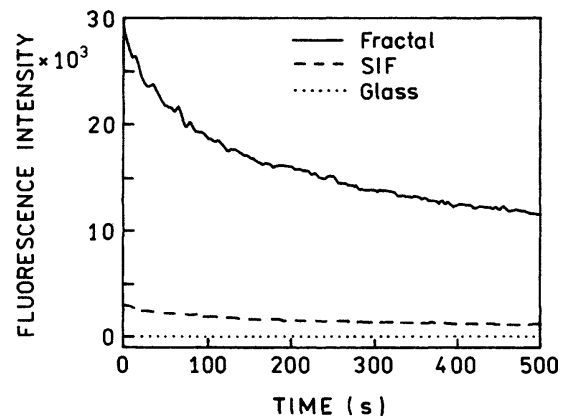

Figure 8. Photostability of FITC-HSA deposited on glass $(\cdots)$, SIF (-- ), and fractal silver (-). The samples were illuminated at $514 \mathrm{~nm}$.

detected from FITC-HSA on SIFs or fractal silver, respectively, relative to quartz, prior to photobleaching.

\section{Summary}

What are the advantages of our approach to making fractal silver structures? One advantage is the absence of solution reagents such as sodium borohydride or citrate to reduce silver ions to metallic islands. Silver deposition is accomplished in pure water. Localization is accomplished by placement of the silver electrodes. Another advantage is that the silver can be freshly generated on demand by an electrical current. Deposition of metallic mobile metals on the surface is presently of intense interest because of the potential applications to nanotechnology. Metal particles can be generated with light ${ }^{28-30}$ and by electroless deposition via galvanic displacement. ${ }^{31}$ Additionally, the fractal silver surfaces result in a partial elimination of selfquenching. As a result it may now be possible to use proteins and DNA, which are heavily overlabeled with fluorophores, as ultrabright reagents themselves for immunoassays or DNA arrays.

One interesting finding here is that metal-enhanced fluorescence appears to be more significant with silver fractal structures, as compared to some of our previous reports. ${ }^{1,2,21,27}$ We believe that this is probably due to the many more opportunities for metal-fluorophore interactions when using 
fractals as compared to lower surface area nanostructures. In addition we show areas of intense fluorescence, "hot-spots", Figures 3 and 4, which is analogous to those observed in SERS, where the relatively lower fluorescence enhancements we have reported to date $1,2,21,27$ can now be attributed to a spatial averaging of the fluorescence emission intensity.

We anticipate these new findings and methods will contribute to the use of metal-enhanced fluorescence in medical diagnostics and biotechnology.

Acknowledgment. This work was supported by the National Institute of Biomedical Imaging and Bioengineering NIHEB00682 and EB-00980 and the National Center for Research Resource, RR-08119.

\section{References and Notes}

(1) Lakowicz, J. R.; Shen, Y.; D’Auria, S.; Malicka, J.; Fang, J.; Gryczynski, Z.; Gryczynski, I. Radiative decay engineering 2. Effects of silver island films on fluorescence intensity, lifetimes, and resonance energy transfer. Anal. Biochem. 2002, 301, 261-277.

(2) Malicka, J.; Gryczynski, I.; Kusba, J.; Shen, Y.; Lakowicz, J. R. Effects of metallic silver particles on resonance energy transfer in labeled bovine serum albumin. Biochem. Biophys. Res. Commun. 2002, 294, 886892.

(3) Lakowicz, J. R.; Shen, B.; Gryczynski, Z.; D’Auria, S.; Gryczynski, I. Intrinsic fluorescence from DNA can be enhanced by metallic particles, Biochem. Biophys. Res. Commun. 2001, 286, 875-879.

(4) Gryczynski, I.; Malicka, J.; Shen, Y.; Gryczynski, Z.; Lakowicz, J. R. Multiphoton excitation of fluorescence near metallic particles: Enhanced and localized excitation. J. Phys. Chem. B 2002, 106, 21912195.

(5) Kummerlen, J.; Leitner, A.; Brunner, H.; Aussenegg, F. R.; Wokaun, A. Enhanced dye fluorescence over silver island films: analysis of the distance dependence. Mol. Phys. 1993, 80 (5), 1031-1046.

(6) Gersten, J.; Nitzan, A. Spectroscopic properties of molecules interacting with small dielectric particles. J. Chem. Phys. 1981, 75 (3), $1139-1152$

(7) Weitz, D. A.; Garoff, S.; Gersten, J. I.; Nitzan, A. The enhancement of Raman scattering, resonance Raman scattering, and fluorescence from molecules absorbed on a rough silver surface. J. Chem. Phys. 1983, 78 (9), $5324-5338$

(8) Chance, R. R.; Prock, A.; Silbey, R. Molecular fluorescence and energy transfer near interfaces. Adv. Chem. Phys. 1978, 37, 1-65.

(9) Lakowicz, J. R. Radiative decay engineering: Biophysical and biomedical applications. Anal. Biochem. 2001, 298, 1-24.

(10) Geddes, C. D.; Cao, H.; Gryczynski, I.; Gryczynski, Z.; Fang, J.; Lakowicz, J. R. Metal-enhanced fluorescence (MEF) due to silver colloids on a planar surface: Potential applications of indocyanine green to in vivo imaging. J. Phys. Chem. A 2003, 107 (18), 3443-3449.

(11) Yakovleva, J.; Davidsson, R.; Lobanova, A.; Bengtsson, M.; Eremin, S.; Laurell, T.; Emneus, J. Microfluidic enzyme immunoassay using silicon microchip with immobilized antibodies and chemiluminescence detection, Anal. Chem. 2002, 74, 2994-3004.

(12) Christodoulides, N.; Tran, M.; Floriano, P. N.; Rodriquez, M.; Goodey, A.; Ali, M.; Neikirk, D.; McDevitt, J. T. A microchip-based multianalyte assay system for the assessment of cardiac risk. Anal. Chem. 2002, 74, 3030-3036.

(13) Verpoorte, E. Microfluidic chips for clinical and forensic analysis. Electrophoresis 2002, 23, 677-712.

(14) Keir, R.; Igata, E.; Arundell, M.; Smith, W. E.; Graham, D.; McHugh, C.; Cooper, J. M. SERRS. In situ substrate formation and improved detection using microfluidics. Anal. Chem. 2002, 74, 1503-1508.

(15) Haynes, C. L.; McFarland, A. D.; Smith, M. T.; Hulteen, J. C.; Van Duyne, R. P. Angle-resolved nanosphere lithography: Manipulation of nanoparticle size, shape, and interparticle spacing. J. Phys. Chem. B 2002 , 106, 1898-1902.

(16) Hua, F.; Cui, T.; Lvov, Y. Lithographic approach to pattern selfassembled nanoparticle multilayers. Langmuir 2002, 18, 6712-6715.

(17) Fleury, V.; Watters, W. A.; Allam, L.; Devers, T. Rapid electroplating of insulators. Nature 2002, 416, 716-719.

(18) Fleischmann, M.; Hendra, P. J.; McQuillan, A. J. Raman spectra of pyridine adsorbed at a silver electrode. Chem. Phys. Lett. 1974, 26 (2), $163-166$.

(19) Kneipp, K.; Kneipp, H.; Itzkan, I.; Dasari, R. R.; Feld, M. S. Surface-enhanced Raman scattering: A new tool for biochemistry spectroscopy. Curr. Sci. 1999, 77 (7), 915-924.

(20) Vo-Dinh, T.; Stokes, D. L.; Griffin, G. D.; Volkan, M.; Kim, U. J.; Simon, M. I. Surface-enhanced Raman scattering (SERS) method and instrumentation for genomics and biomedical analysis. J. Raman Spectrosc. 1999, 30, 785-793.

(21) Lakowicz, J. R.; Malicka, J.; D’Auria, S.; Gryczynski, I. Release of the self-quenching of fluorescence near silver metallic surfaces. Anal. Biochem., in press.

(22) Laczko, G.; Gryczynski, I.; Gryczynski, Z.; Wiczk, W.; Malak, H.; Lakowicz, J. R. A GHz frequency-domain fluorometer. Rev. Sci. Instrum. 1990, 61, 2331-2337.

(23) Lakowicz, J. R.; Laczko, G.; Cherek, H.; Gratton, E.; Limkeman, M. Analysis of fluorescence decay kinetics from variable-frequency phase shift and modulation data. Biophys. J. 1994, 46, 463-477.

(24) Sokolov, K.; Chumanov, G.; Cotton, T. M. Enhancement of molecular fluorescence near the surface of colloidal metal films. Anal. Chem 1998, 70, 3898-3905.

(25) Nie, S.; Emory, S. R. Probing single molecules and single nanoparticles by surface-enhanced Raman scattering. Science 1997, 275 (21 February), 1102-1106.

(26) Kneipp, K.; Kneipp, H.; Bhaskaran Kartha, V.; Manoharan, R. Deinum, G.; Itzkan, I.; Dasari, R. R.; Feld, M. S. Detection and identification of a single DNA base molecule using Surface-Enhanced Raman Scattering (SERS). Phys. Rev. E 1998, 57 (6), R6281-R6284.

(27) Malicka, J.; Gryczynski, I.; Gryczynski, Z.; Lakowicz, J. R. Effects of fluorophore-to-silver distance on the emission of cyanine-dye labeled oligonucleotides. Anal. Biochem. 2003, 315, 57-66.

(28) Abid, J. P.; Wark, A. W.; Brevet, P. F.; Girault, H. H. Preparation of silver nanoparticles in solution from a silver salt by laser irradiation. Chem. Commun. 2002, 7, 792-793.

(29) Bjerneld, E. J.; Murty, K. V. G. K.; Prikulis, J.; Käll, M. Laserinduced growth of $\mathrm{Ag}$ nanoparticles from aqueous solutions. Chem. Phys. Chem. 2002, 116-119.

(30) Pastoriza-Santos, I.; Serra-Rodriguez, C.; Liz-Marzan, L. M. Selfassembly of silver particles monolayers on glass from $\mathrm{Ag}^{+}$solutions in DMF. J. Colloid Interface Sci. 2000, 221, 236-241.

(31) Porter, L. A.; Choi, H. C.; Ribbe, A. E.; Buriak, J. M. Controlled electroless deposition of noble metal nanoparticle films on germanium surfaces. Nano Lett. 2002, 2 (10), 1067-1071. 